\title{
Core Topics in Critical Care Medicine
}

\author{
Fang Gao Smith (Editor). Joyce Yeung (Associate Editor). Cambridge University Press, \\ 2010. ISBN 978-0-521-89774-7
}

\author{
Stephan Langevin, MD
}

Received: 13 July 2011/Accepted: 31 August 2011/Published online: 30 September 2011

(C) Canadian Anesthesiologists' Society 2011

The major contributors of this first edition textbook in critical care are from Birmingham, United Kingdom, and as stated in the Preface, their target audience is primarily trainees from all medical specialities undertaking subspecialty training in critical care medicine as well as critical care clinicians and allied health professionals working in the intensive care environment.

The textbook includes the following four sections which cover 48 topics in critical care medicine: "Specific Features of Critical Care Medicine", "Systemic Disorders and Management", "Organ Dysfunction and Management", and "Examinations". Each topic is developed with an introduction, a core portion, and a key points section, and each concludes with a "Further Reading" section wherein the authors recommend a list of two to ten references. The variety of suggested references ranges from textbooks and websites to features and review articles and guidelines from medical societies, boards, or associations. Each topic is covered within five to 18 pages, and as some topics share common characteristics, they could easily be incorporated within a different section in the textbook (e.g., the topics, "Acid-base abnormalities" and "Fluid and electrolyte disorders", lend themselves more to Section I, "Specific Features of Critical Care Medicine" than to Section II, "Systemic Disorders and Management").

As implied by the title, this textbook does not elaborate on each topic of critical care, rather, its focus is strictly on the essentials of each issue. For instance, the topic, "Trauma and burns", is covered in eight pages, and the topic, "Traumatic brain injury", is covered in nine pages.

S. Langevin, MD ( $ه)$

Université Laval, Quebec City, QC, Canada

e-mail: slangevin@videotron.ca
For non-initiated medical students and trainees, this text is truly on overview of the many challenges in critical care medicine. The book features clear tables which contrast effectively against the text, and, for the most part, there are good-quality photos and figures, although some are photocopies which may have been enhanced (Chapters 24, 39). The final section, "Examinations", may not be comprehensive enough to help trainees in critical care be successful in their quest to obtain the UK Intercollegiate Diploma in Intensive Care Medicine (DICM) or the European Diploma in Intensive Care Medicine (EDIC). As a final point, the abbreviations and the index sections are exhaustive and complete.

With websites such as the European Society of Critical Care Medicine (www.esicm.org) and the Society of Critical Care Medicine (www.sccm.org) as well as the many review papers published every year by these societies, medical associations, or foundations in their various journals, it is difficult to imagine that a second edition of a textbook giving an overview of topics in critical care medicine would be published or have a place in our library. The field of critical care medicine is crowded with many review papers, recommendations, and guidelines. Another important issue these days is the fact that students and trainees in the various medical specialties are more clued in and eager to study on the internet rather than with textbooks. To appeal to trainees, the material must be accessible on the web. Additionally, I suspect this textbook lacks sufficient appeal for trainees to purchase it. Despite these shortcomings, the contributors to this textbook put forth ample effort to simplify and introduce the field of critical care medicine to young medical students.

Competing interests None declared. 